\title{
Low-contrast pre-coronagraph for extra contrast of dark-hole
}

\author{
Jun Nishikawa ${ }^{1,2,3}$ Masahito Oya $^{4,1}$ Naoshi Murakami $^{5}$ \\ Motohide Tamura ${ }^{6,1,3}$ Takashi Kurokawa $a^{1,7}$ Yosuke Tanaka $^{7}$ and \\ Takayuki Kotani ${ }^{3,1}$
${ }^{1}$ National Astronomical Observatory of Japan, Extrasolar Planet Detection Project Office, 2-21-1 Osawa, Mitaka, Tokyo, Japan, 181-8588 email: jun.nishikawa@nao.ac.jp
${ }^{2}$ SOKENDAI (Graduate University for Advanced Studies), Faculty of Physical Sciences, 2-21-1 Osawa, Mitaka, Tokyo, Japan, 181-8588
${ }^{3}$ National Institute of Natural Sciences, Astrobiology Center, 2-21-1 Osawa, Mitaka, Tokyo, Japan, 181-8588
${ }^{4}$ Nihon University, Graduate School of Physics, Surugadai 1-8-14, Chiyoda, Tokyo, Japan, 101-8308
${ }^{5}$ Hokkaido University, Fuculty of Engineering, Kita 13 Nishi 8, Kita-Ku, Sapporo, Hokkaido, Japan, 060-8628
${ }^{6}$ The University of Tokyo, Graduate School of Science, Department of Astronomy, 7-3-1 Hongo, Bunkyo-ku, Tokyo, Japan, 113-0033
${ }^{7}$ Tokyo University of Agriculture and Technology, Graduate School of Engineering, \\ Koganei, Tokyo, Japan, 184-8588
}

\begin{abstract}
We propose a low-contrast pre-coronagraph that can provide additional dark-hole contrast to a main coronagraph.
\end{abstract}

Keywords. instrumentation: adaptive optics, techniques: interferometric.

\section{Pre-coronagraph under dark-hole control}

The low-contrast pre-coronagraph (LPC) is a new style of the unbalanced nulling interferometer (UNI) which was developed for precise wavefront control (Nishikawa et al. 2008). The LPC is used in the four-stage coronagraph system: the first deformable mirror $(\mathrm{DM})$, the LPC, the second DM, and the main coronagraph, to obtain an additional contrast to the main coronagraph. Originally a wavefront sensor was used around the UNI (LPC) and we characterized it using a four-quadrant phase mask coronagraph (Kobayashi, et al. 2012). Recently we have found that the two deformable mirrors in the system can be controlled by the dark-hole algorithm with a final focal-plane detector if we use two steps. First, the control is made by the first DM with a normal mask at the pre-coronagraph and without a mask at the main coronagraph. Second, the control is made by both two DMs to produce the circular dark hole with a low-contrast mask exchanged at the pre-coronagraph and with a normal mask at the main coronagraph.

\section{References}

Nishikawa, J., Abe, L., Murakami, M., \& Kotani, T. 2008, A\&3A, 489, 1389

Kobayashi, T., Nishikawa, J., Tanaka, Y., Kurokawa, T., Kashiwagi, K., Murakmai, N., Baba, N., \& Hashimoto, N. 2012, Proc. SPIE, 8421, 84213D 